Electronic Supplementary Information (ESI)

\title{
Rapid Fabrication of Composite Hydrogel Microfibers for Weavable and Sustainable Antibacterial Applications
}

Chuntao Chen ${ }^{a}$, Ting Zhang ${ }^{b}$, Beibei Dai ${ }^{a}$, Heng Zhang ${ }^{a}$, Xiao Chen ${ }^{a}$, Jiazhi Yang ${ }^{a}$, Jian Liu $^{b^{*}}$ and

Dongping $\operatorname{Sun}^{a^{*}}$

${ }^{a}$ Institute of Chemicobiology and Functional Materials, School of Chemical Engineering, Nanjing University of Science and Technology, 200 Xiao Ling Wei Street, Nanjing, Jiangsu Province, China.

${ }^{b}$ Institute of Functional Nano and Soft Materials (FUNSOM), Soochow University, 199 Ren Ai Road, Suzhou Industrial Park, Suzhou, Jiangsu Province, China.

*Tel: 0258431 5079. Fax: 0258443 1939. E-mail: sundpe301@163.com (Dongping Sun); jliu@suda.edu.cn (Jian Liu).

Number of pages: 9

Number figures: 12

Number tables: 0 


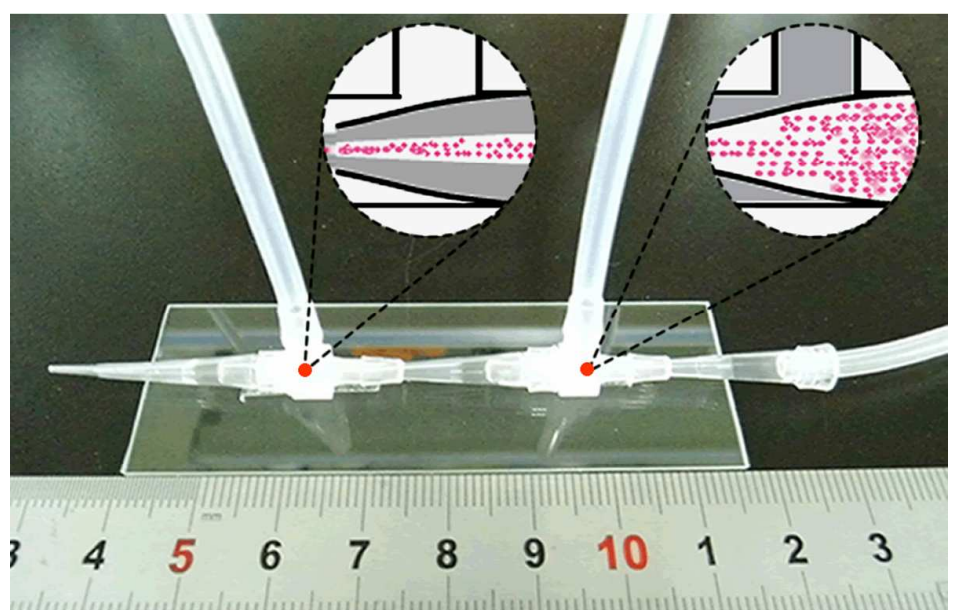

Figure S1. Optical photograph of the double-coaxial laminar flow microfluidic device.

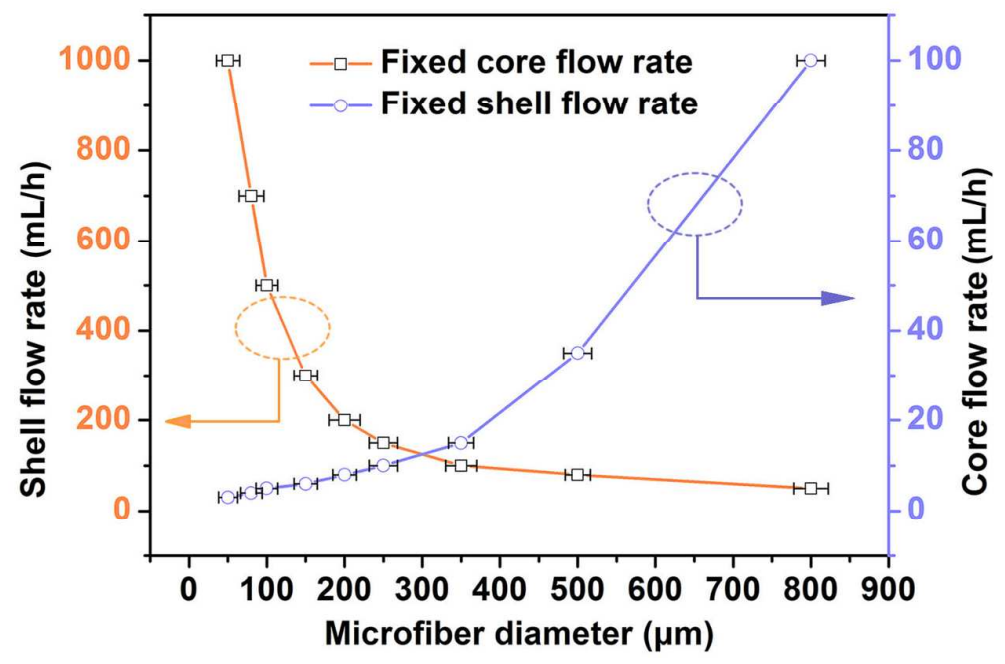

Figure S2. The relationships of the shell flow rate or the core flow rate on the fiber diameter. Error bar: standard deviation $(n=3)$. 

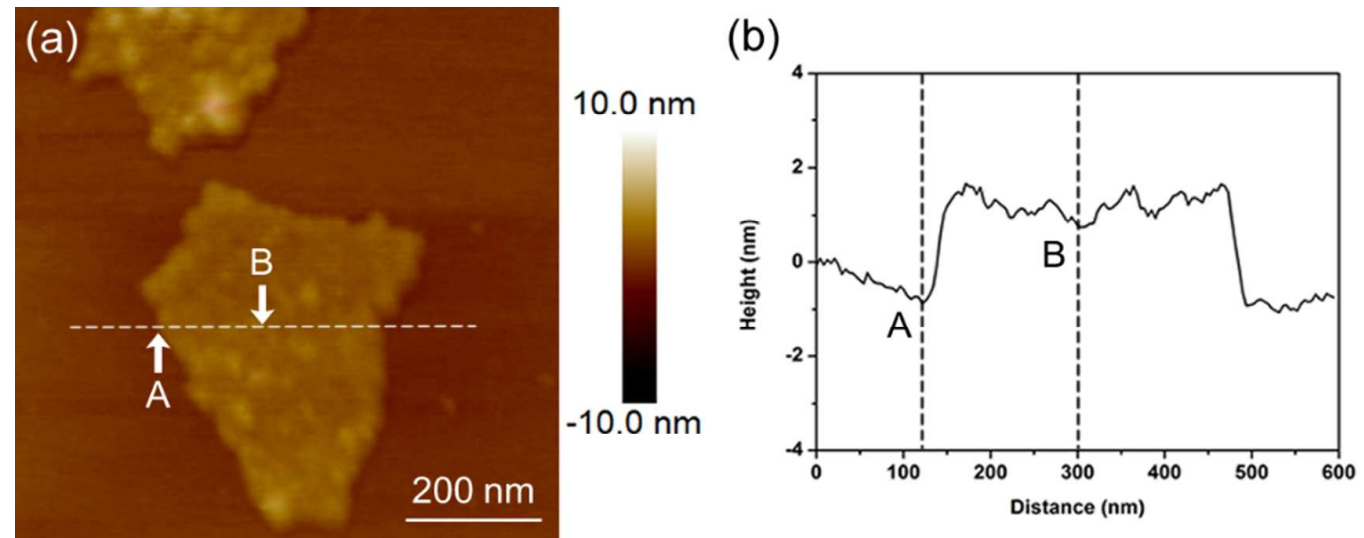

Figure S3. (a) AFM image of GO sheets deposited on a freshly cleaved mica surface; (b) The heights of GO sheets were uniformly around $2 \mathrm{~nm}$.
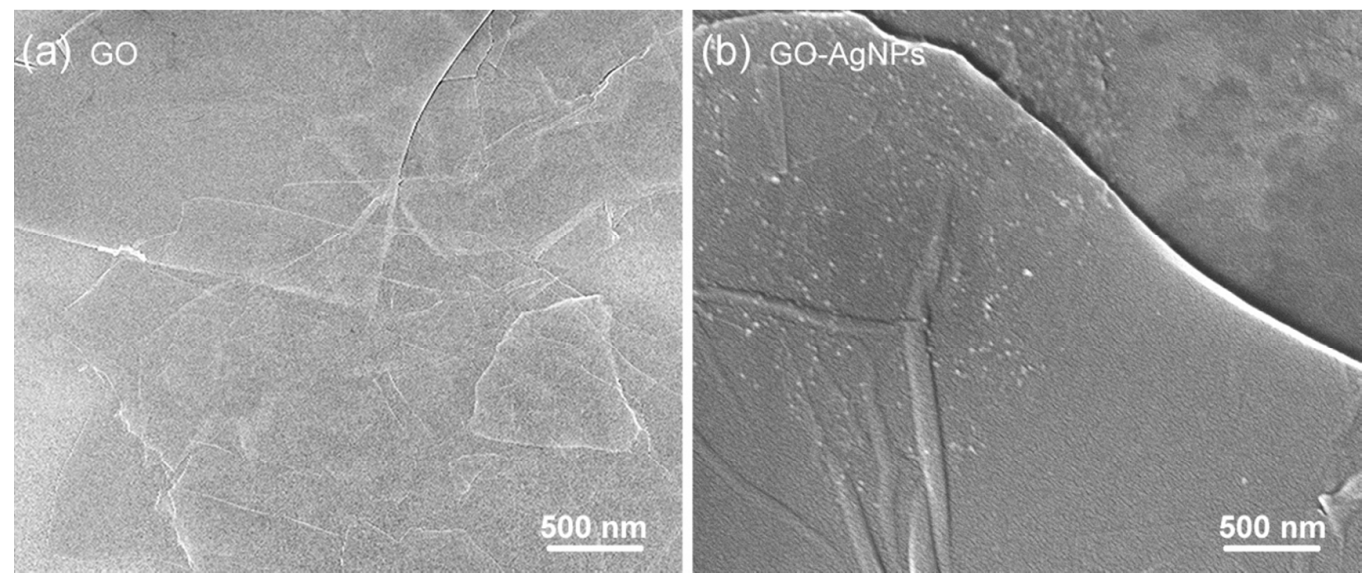

Figure S4. SEM images of GO (a) and GO-AgNPs (b). 


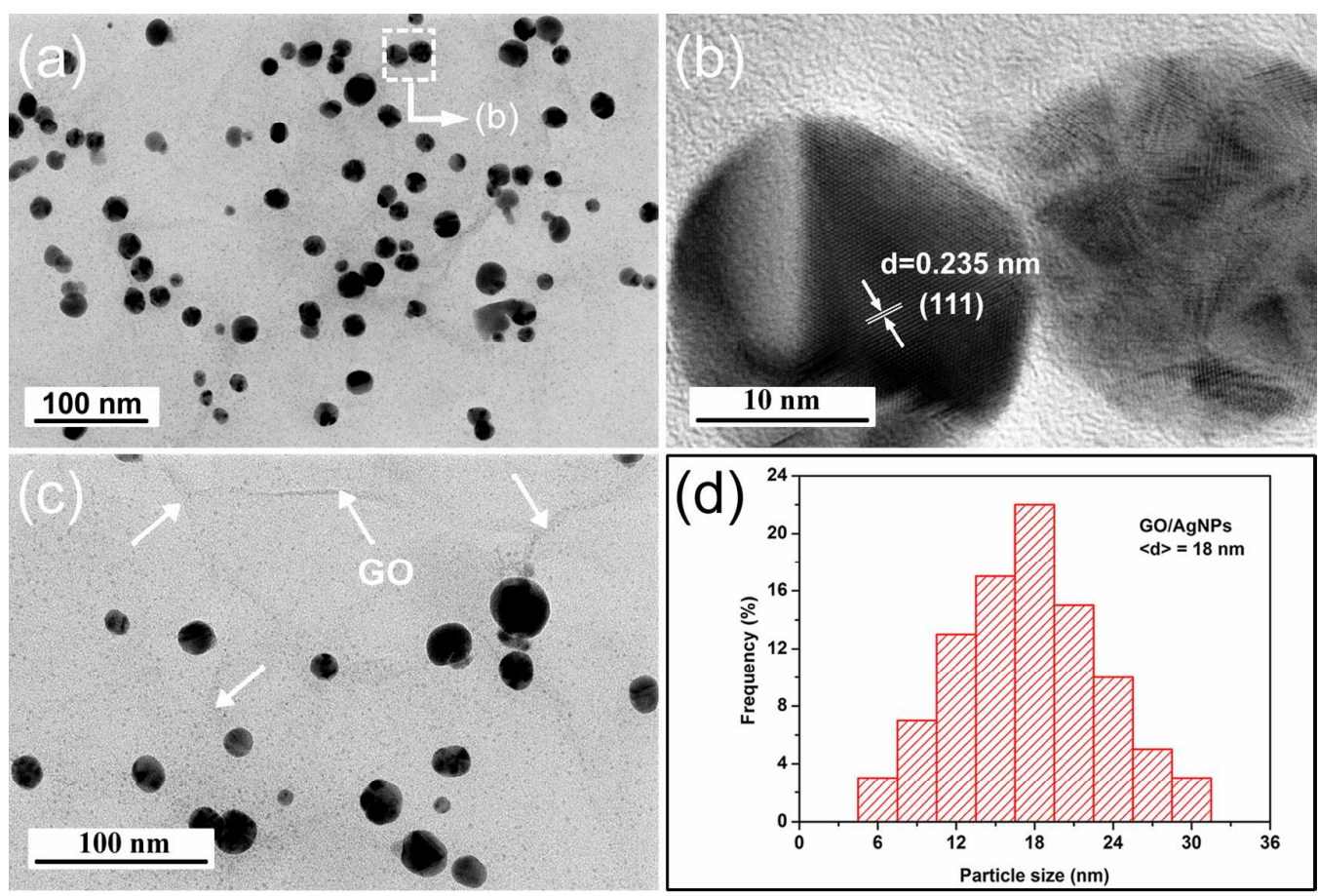

Figure S5. (a, b, c) TEM images of GO-AgNPs and (d) the average size of AgNPs measured in the TEM images. 

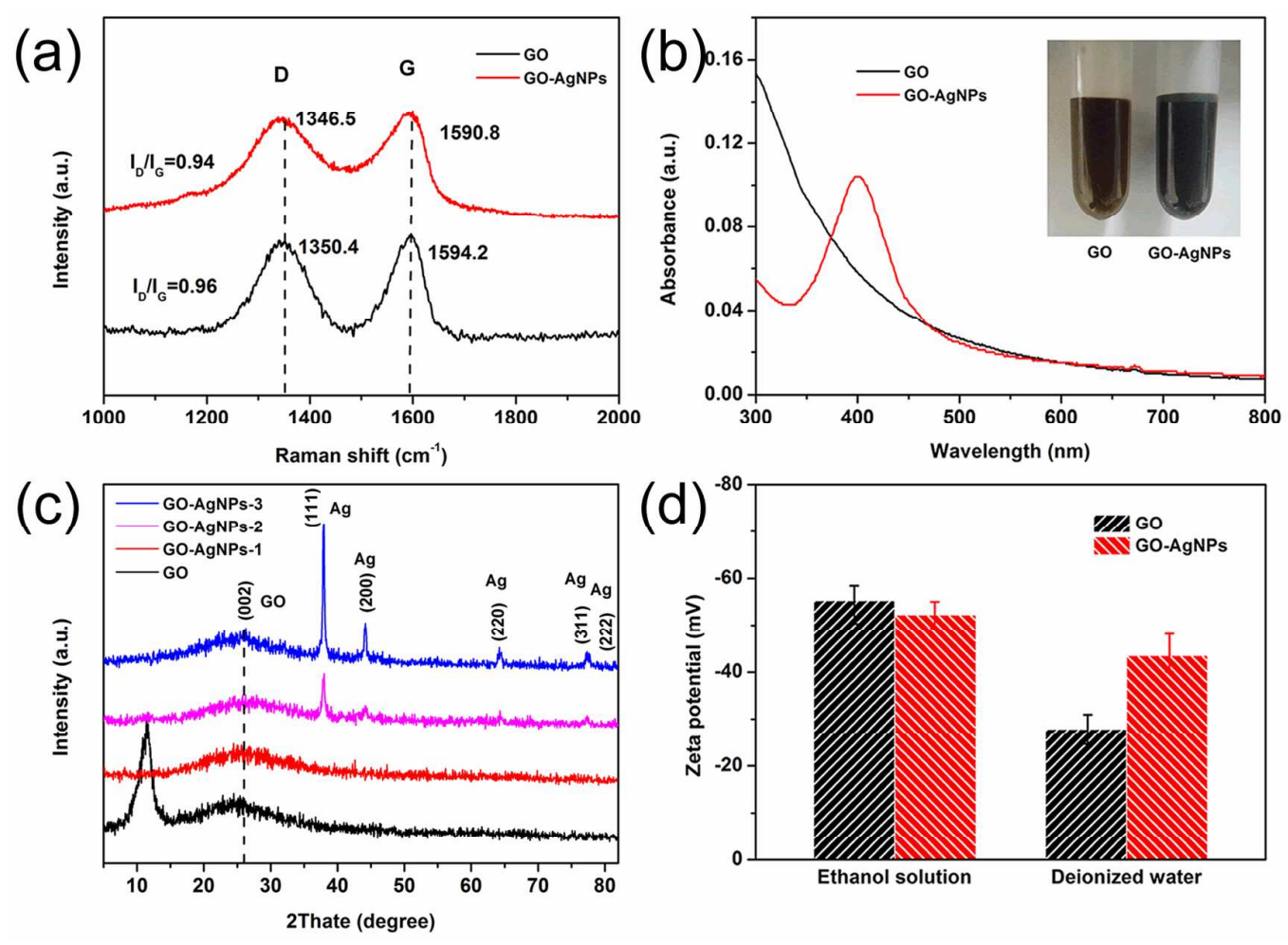

Figure S6. (a) Raman spectra of GO and GO-AgNPs; (b) UV-Vis absorption spectra of GO and GO-AgNPs; (c) XRD analysis of GO and GO-AgNPs with different ratios of Ag and grapheme; (d) Zeta potential of GO and GO/AgNPs in deionized water and ethanol solution. 
GO-AgNPs were prepared by in situ reduction of $\mathrm{Ag}^{+}$ions at graphite oxide (GO) surface (Figure S3 and S4) in a solution of sodium citrate. The average size of AgNPs was about $18 \mathrm{~nm}$ (Figure S5d). The averaged zeta potential of AgNPs in water and ethanol are $-52.3 \mathrm{mV}$ and $-43.5 \mathrm{mV}$ (Figure S6d). ${ }^{1}$ The measured lattice fringe spacing of $0.235 \mathrm{~nm}$ in these nanoparticles (Figure S5b) was corresponding to the face-centered cubic lattice of $\mathrm{Ag}$ nanocrystals. ${ }^{2}$ Figure S6c was the X-ray diffraction (XRD) pattern of the GO-AgNPs composite. Except for the diffraction peak of GO concerted at $2 \theta=10.4^{\circ}$, the peaks at $38.1^{\circ}, 44.2^{\circ}, 64.5^{\circ}, 77.5^{\circ}$ and $81.2^{\circ}$ can be respectively indexed to the (111), (200), (220), (311) and (222) diffractions of

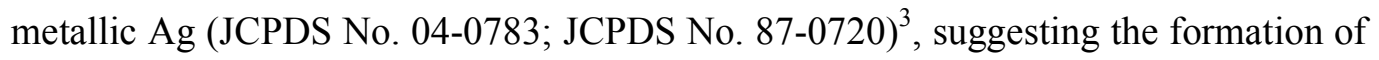
metallic Ag particles. ${ }^{4}$ Figure S6a showed the Raman spectra of GO and GO-AgNPs. For the spectra of GO, the $\mathrm{G}$ band displayed at $1597.2 \mathrm{~cm}^{-1}$, the D band at $1358.4 \mathrm{~cm}^{-1}$, the ration of $\mathrm{D} / \mathrm{G}$ intensity of GO was 0.96 . The Raman spectrum of GO-AgNPs also displayed $\mathrm{G}$ and $\mathrm{D}$ bands, at $1595.8 \mathrm{~cm}^{-1}$ and $1356.9 \mathrm{~cm}^{-1}$ respectively, the ration of $\mathrm{D} / \mathrm{G}$ intensity was 0.94 , as a result of decreasing the defects of GO to some extent of reduction. $^{5}$
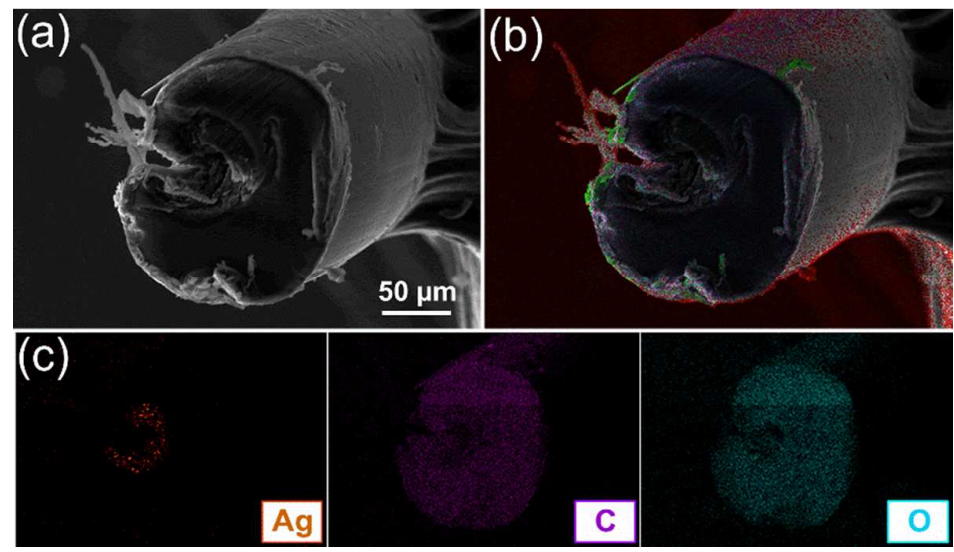

Figure S7. The EDX mapping of the GO-AgNPs/BC microfiber. 


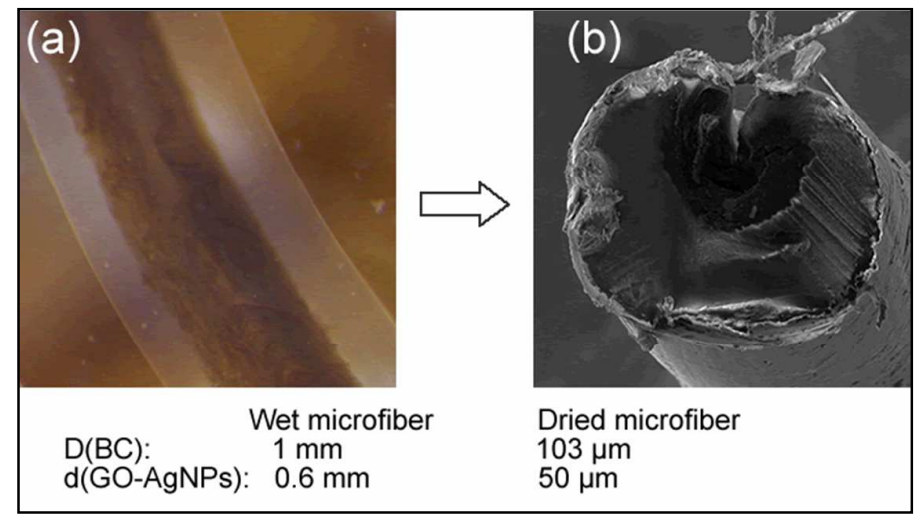

Figure S8. The core/shell GO-AgNPs/BC microfiber turn from wet to dried.

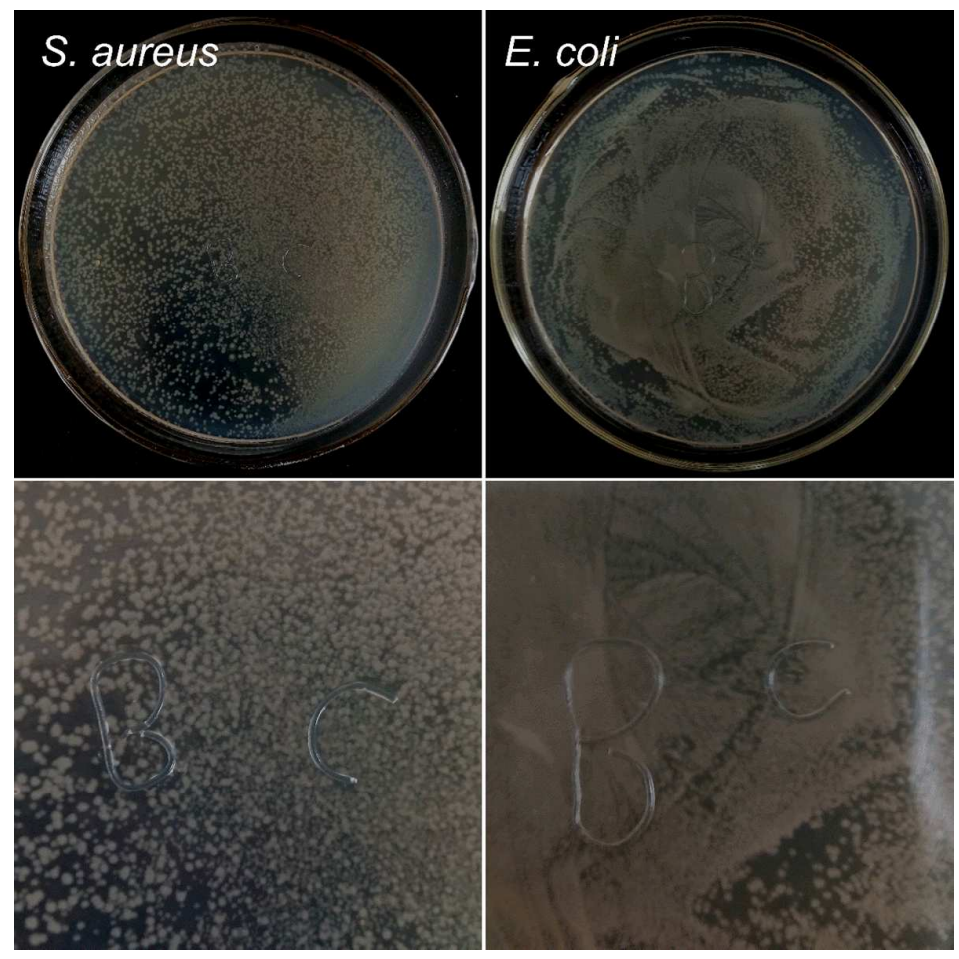

Figure S9. The antibacterial performance of $\mathrm{BC}$ hydrogel microfibers against Gram negative bacterial strains, E. coli (right) and Gram positive bacterial strains, S. aureus (left). 


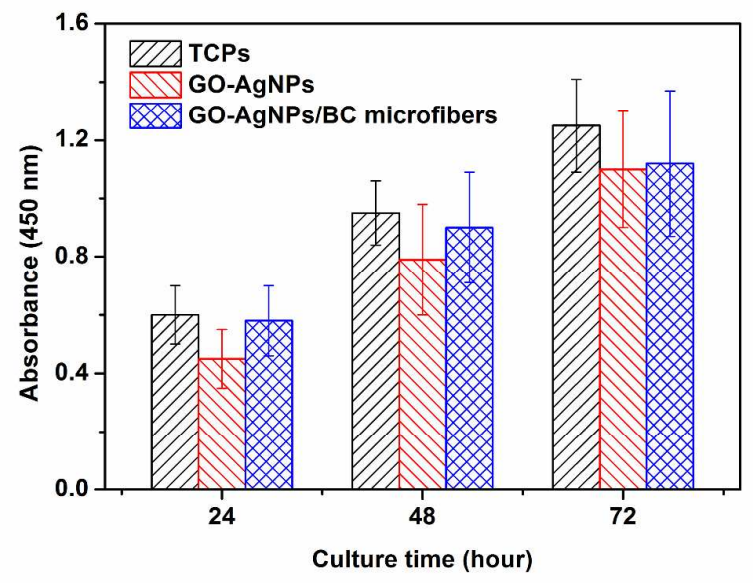

Figure S10. Relative viabilities of L02 cells cultured in the media at different culture time containing $10 \mu \mathrm{g} \mathrm{mL} \mathrm{m}^{-1} \mathrm{GO}-\mathrm{AgNPs} / \mathrm{BC}$ microfibers, Error bars: standard deviations $(n=3)$.

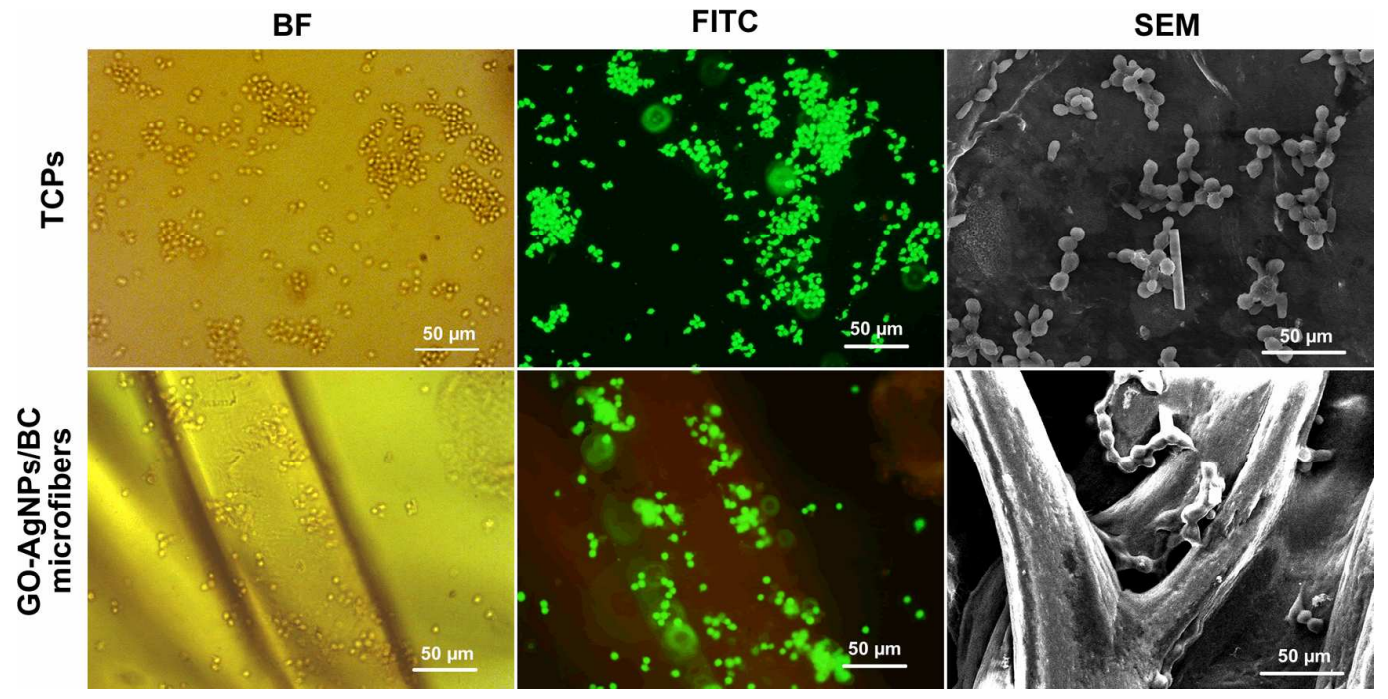

Figure S11. Bright field (left), fluorescence images (middle) of L02 cellular skeleton structure and SEM images (right) of L02 cells cultured on the GO-AgNPs/BC microfibers and TCPs. 


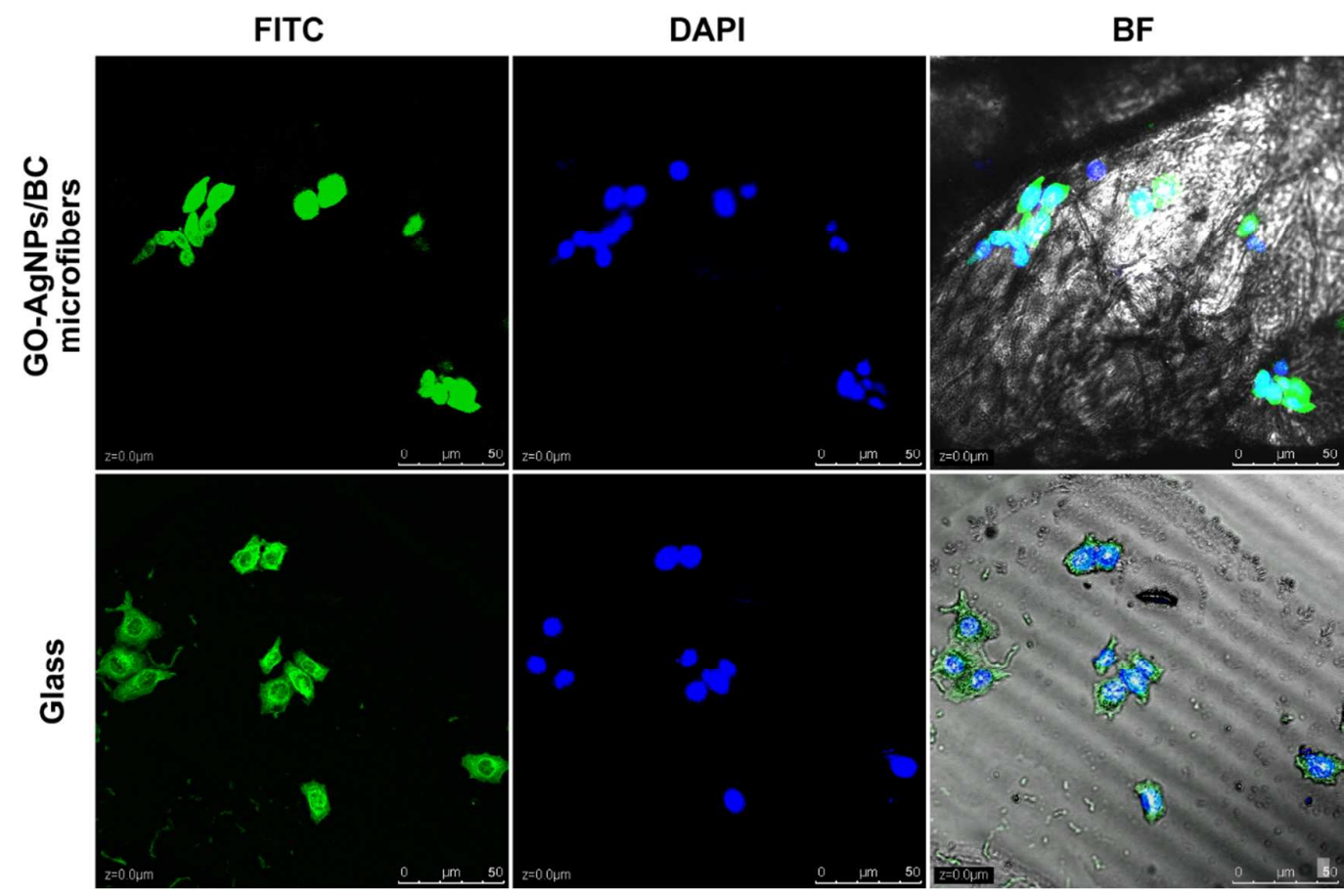

Figure S12. Fluorescence images of L02 cellular skeleton structure on the GO-AgNPs/BC microfibers and glass.

1. Lu, L.; Liu, J.; Hu, Y.; Zhang, Y.; Chen, W. Graphene-stabilized silver nanoparticle electrochemical electrode for actuator design. Advanced materials 2013, 25, (9), 1270-1274.

2. Tang, X. Z.; Cao, Z.; Zhang, H. B.; Liu, J.; Yu, Z. Z. Growth of silver nanocrystals on graphene by simultaneous reduction of graphene oxide and silver ions with a rapid and efficient one-step approach. Chemical communications 2011, 47, (11), 3084-3086.

3. Sureshkumar, M.; Siswanto, D. Y.; Lee, C.-K. Magnetic antimicrobial nanocomposite based on bacterial cellulose and silver nanoparticles. Journal of Materials Chemistry 2010, 20, (33), 6948-6955.

4. Ma, J.; Zhang, J.; Xiong, Z.; Yong, Y.; Zhao, X. S. Preparation, characterization and antibacterial properties of silver-modified graphene oxide. Journal of Materials Chemistry 2011, 21, (10), 3350-3352.

5. Li, C.; Wang, X.; Chen, F.; Zhang, C.; Zhi, X.; Wang, K.; Cui, D. The antifungal activity of graphene oxide-silver nanocomposites. Biomaterials 2013, 34, (15), 3882-3890. 